\title{
Impact of Screw Diameter and Length on Pedicle Screw Fixation Strength in Osteoporotic Vertebrae: A Finite Element Analysis
}

\author{
Keitaro Matsukawa ${ }^{1}$, Yoshiyuki Yato ${ }^{1}$, Hideaki Imabayashi ${ }^{2}$ \\ ${ }^{1}$ Department of Orthopaedic Surgery, National Hospital Organization, Murayama Medical Center, Musashimurayama, Japan \\ ${ }^{2}$ Department of Orthopaedic Surgery, Tokyo Saiseikai Central Hospital, Tokyo, Japan
}

\begin{abstract}
Study Design: Biomechanical study.
Purpose: To quantitatively investigate the effect of screw size on screw fixation in osteoporotic vertebrae with finite element analysis (FEA)

Overview of Literature: Osteoporosis poses a challenge in spinal instrumentation; however, the selection of screw size is directly related to fixation and is closely dependent on each surgeon's experience and preference.

Methods: Total 1,200 nonlinear FEA with various screw diameters $(4.5-7.5 \mathrm{~mm})$ and lengths $(30-50 \mathrm{~mm})$ were performed on 25 patients (seven men and 18 women; mean age, $75.2 \pm 10.8$ years) with osteoporosis. The axial pullout strength, and the vertebral fixation strength of a paired-screw construct against flexion, extension, lateral bending, and axial rotation were examined. Thereafter, we calculated the equivalent stress of the bone-screw interface during nondestructive loading. Then, using diameter parameters (screw diameter or screw fitness in the pedicle [\%fill]), and length parameters (screw length or screw depth in the vertebral body [\%length]), multiple regression analyses were performed in order to evaluate the factors affecting various fixations.

Results: Larger diameter and longer screws significantly increased the pullout strength and vertebral fixation strength; further, they decreased the equivalent stress around the screws. Multiple regression analyses showed that the actual screw diameter and \%length were factors that had a stronger effect on the fixation strength than \% fill and the actual screw length. Screw diameter had a greater effect on the resistance to screw pullout and flexion and extension loading $\beta=0.38-0.43, p<0.01)$; while the \%length had a greater effect on resistance to lateral bending and axial rotation loading $(\beta=0.25-0.36, p<0.01)$ as well as mechanical stress of the bone-screw interface $(\beta=-0.42, p<0.01)$.

Conclusions: The screw size should be determined based on the biomechanical behavior of the screws, type of mechanical force applied on the corresponding vertebra, and anatomical limitations.
\end{abstract}

Keywords: Finite element analysis; Pedicle screws; Osteoporosis; Screw size; Fixation strength

\section{Introduction}

Pedicle screw fixation has become the most reliable tech- nique for managing several spinal pathologies that enable the stabilization of spinal segments and facilitate fusion. The anchoring ability of pedicle screws is influenced by the

Received Jul 9, 2020; Revised Aug 22, 2020; Accepted Aug 22, 2020

Corresponding author: Keitaro Matsukawa

Department of Orthopaedic Surgery, National Hospital Organization, Murayama Medical Center, 2-37-1 Gakuen, Musashimurayama, Tokyo 208-0011, Japan

Tel: +81-42-561-1221, Fax: +81-42-564-2210, E-mail: keitaro197897@hotmail.com 
following three major factors: bone properties [1], screw design and size [2-4], and insertion technique [5]. Among these, the vertebral bone quality is reported to be the most critical factor $[1,6,7]$, indicating that osteoporosis poses a challenge owing to fragile bone characteristics, often resulting in screw loosening, correction loss, fusion failure, and revision surgery. In addition to intervention with osteoporosis therapeutics, augmentation of the screw hole with cement or hydroxyapatite is another method that can be used to enhance the integrity of the bone-screw interface. Another method involves increasing the contact area of the bone-screw interface via design optimization and pedicle screw size. However, despite the development of technologies, such as navigation systems and robotic surgeries, that enable accurate screw placement, the selection of screw size and design, factors that are directly related to fixation, heavily depends on each surgeon's experience and preference. In some cases, it may not be possible to select the optimal screw size because of the anatomical risk of neurovascular injuries and pedicle fracture.

Several biomechanical studies have assessed the mechanical properties of screws for improved fixation. The quality and quantity of the bone in the screw thread play a crucial role in withstanding the physiological load and screw pullout; therefore, the use of a larger outer diameter, conical core, and longer screws has been recommended $[4,6,8]$. Many trials have shown that increases in the outer diameter to enable fixation in the pedicle improved the fixation performance $[2,9,10]$. However, the application of larger diameter screws to osteoporotic vertebrae remains controversial because of thinner and weaker cortical layer of the pedicle, potentially resulting in less fixation [11]. Lai et al. [12] concluded that for osteoporotic vertebrae, larger diameter screws improved fixation only immediately following implantation as compared to smaller diameter screws; however, there was no long-term improvement. Possible reasons for these differences include an insufficient sample size, wide individual variation in the bone quality and the level tested, mismatched screw properties, and differences in the evaluation method, leading to biases in the study and misinterpretation of the results. In order to eliminate such biases, the material and experimental environment should be strictly controlled for assessing the true differences among screws of various sizes.

Finite element (FE) analysis is a useful method and is increasingly being used in the field of spinal surgery
[3,4,10,13-15], allowing fair comparisons via flexible modification of various parameters under the same environmental conditions. With spinal instrumentation surgery being performed more commonly in osteoporosis patients, owing to population aging, the present study aimed to quantitatively investigate the effect of screw size on screw fixation in osteoporotic vertebrae.

\section{Materials and Methods}

\section{Subjects}

This study was performed after obtaining the institutional review board approval from the Research Ethics Committee of Murayama Medical Center (ID: 12-10). Informed consent was obtained from all individual participants included in the study.

The computed tomography (CT) scan data of 25 osteoporotic patients (seven men and 18 women; mean age, $75.2 \pm 10.8$ years; mean T-score, $-2.8 \pm 0.3$ ) who underwent surgery for lumbar degenerative disorders were analyzed. Osteoporosis was defined as T-score $\leq-2.5$ at the lumbar spine assessed using dual-energy X-ray absorptiometry (DEXA). Patients with vertebral fracture, infection, metastasis, vertebral malformation, and a previous history of surgery of the lower lumbar spine were excluded. There were 18 patients with degenerative spondylolisthesis and seven with canal stenosis. Each patient preoperatively underwent CT with a slice thickness of $1 \mathrm{~mm}$ and was subjected to bone mineral density (BMD) assessment using DEXA for the femoral neck and the lumbar spine.

\section{Finite element models}

FE model construction and biomechanical analyses were performed using Mechanical Finder software (ver. 6.2, extended edition; Research Center of Computational Mechanics, Tokyo, Japan) as per a previously reported testing protocol $[14,15]$. Three-dimensional FE models of the L4 vertebra were prepared as per the individual CT data. The inhomogeneity of bone density and mechanical properties were assessed using the corresponding Hounsfield unit (HU) values in $\mathrm{CT}$ and the equations described by Keyak et al. [16]. FE models of pedicle screws (SOLERA Spinal System; Medtronic, Memphis, TN, USA) that were designed with the cortical thread at the proximal shaft and cancellous thread at the screw tip, were developed using 
high-resolution micro-CT and were assigned the material properties of cobalt chromium alloy for the screw heads and titanium alloy for the screw shaft. Each bone model was bilaterally placed with pedicle screws with different diameters $(4.5 \mathrm{~mm}, 5.5 \mathrm{~mm}, 6.5 \mathrm{~mm}$, and 7.5 $\mathrm{mm}$ ) and lengths $(30 \mathrm{~mm}, 35 \mathrm{~mm}, 40 \mathrm{~mm}, 45 \mathrm{~mm}$, and $50 \mathrm{~mm}$ ) with the technique of Weinstein et al. [17]. All the screws were in an appropriate position without any cortical breach or penetration into the anterior vertebral cortex. These FE models were then divided into 800,000$1,000,000$ tetrahedral solid elements with a size of $0.5-1$ $\mathrm{mm}$ in order to simulate the smooth surface of the models. Further, the bone-screw interface characteristics were set as contact conditions and the friction coefficient was set to zero [3]. The computer solution time per analysis was 12-36 hours, and total 1,200 nonlinear FE analyses with various screw size and biomechanical testing protocols were performed on models of the 25 individuals.

\section{Anatomical evaluation}

The fitness of screws of each size was assessed by calculating the percent fill (\%fill) and percent length (\%length) (Fig. 1). The \%fill was calculated as the ratio of the outer screw diameter to the width of the minor endosteal in the smallest coronal section of the pedicle perpendicular to

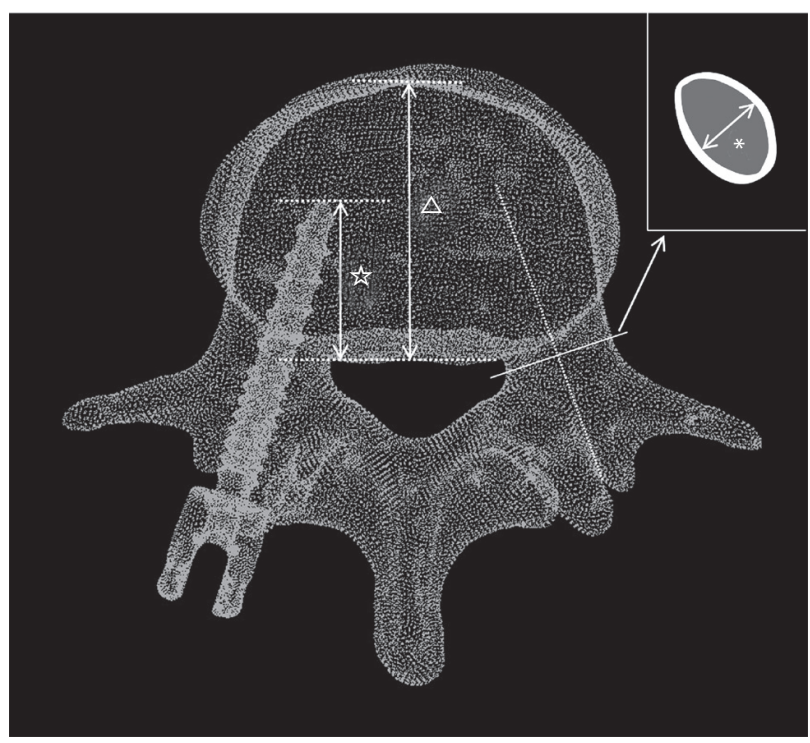

Fig. 1. Percent fill and percent length. Percent fill was defined as the ratio of the outer screw diameter to the minor endosteal width in the smallest coronal section of the pedicle which is perpendicular to the pedicular axis $\left({ }^{*}\right)$. Percent length was defined as the ratio of the screw length within the vertebral body $(\boldsymbol{w})$ to the anteroposterior diameter of the vertebral body $(\Delta)$ in the axial plane. the pedicular axis [18]. Further, the \%length was defined as the ratio of the screw length within the vertebral body to the anteroposterior diameter of the vertebral body [14]. The regional BMD of the center of the pedicle and the vertebral body was calculated using the $\mathrm{HU}$ values on CT by setting an oval-shaped range inside the cortical shell in the axial plane [14].

\section{Boundary and loading conditions}

First, in order to evaluate the pullout strength, the superior and inferior surfaces of the vertebral body were completely fixed. Thereafter, a pullout force was gradually applied to the screw head along the screw axis in increments of $20 \mathrm{~N}$. Screw head displacement was measured, and the pullout strength was defined as the load at the inflexion point of the load-displacement curve.

Then, to evaluate the vertebral fixation strength with paired-screw construct, the bilateral screw heads were fixed completely. Thereafter, an incremental loading rate of $20 \mathrm{~N}$ was applied to the vertebral body surface in order to simulate flexion, extension, lateral bending, and axial rotation loads as per the reported methods (Fig. 2). In destructive loading, the displacement was measured from the average displacement of the entire vertebra; the ultimate failure loads were defined as the load at the inflexion point of the load-displacement curve. Vertebral fixation strength $(\mathrm{N} / \mathrm{mm})$ was defined as the slope of the line fitting the load-displacement curve till the time of ultimate failure load.

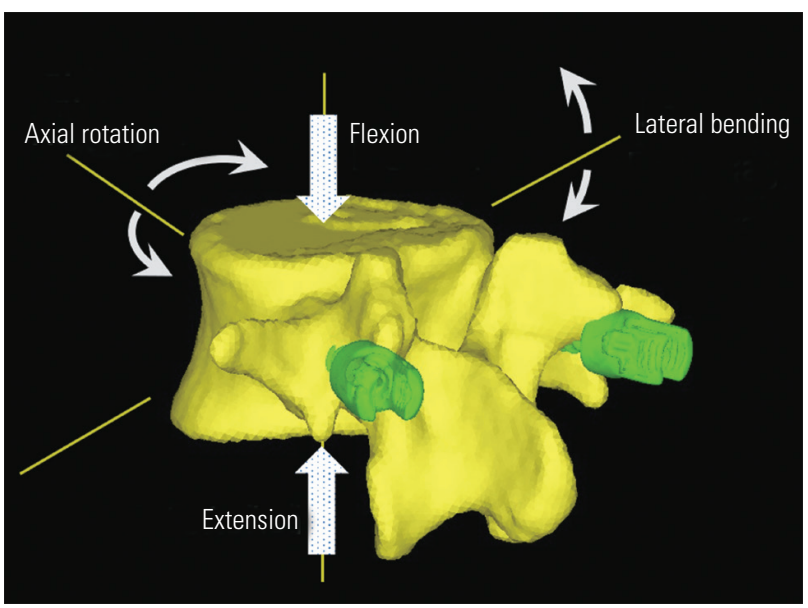

Fig. 2. Evaluation of vertebral fixation strength by paired pedicle screws. IIlustration of loading conditions for flexion, extension, lateral bending, and axial rotation. 
Finally, under the same boundary condition as that used for vertebral fixation strength, a 200-N downward force was applied on the superior surface of the vertebral body after the nondestructive testing protocol that was described by Chen et al. [13]. We evaluated the mean Drucker-Prager equivalent stress of the bone-screw interface.

\section{Statistical analyses}

All the results are presented as mean \pm standard deviation values. Pearson's correlation coefficient, repeatedmeasures analysis of variance, and Turkey's significant difference multiple comparison tests were used for the statistical analyses. The BMD parameters (measured with DEXA or HU values on CT), diameter parameters (screw diameter or \%fill), and length parameters (screw length or \%length) were used for multiple regression analyses with a best-subset selection procedure to evaluate the factors that affected the various fixation strengths. JMP ver. 13.0 (SAS Institute Inc., Cary, NC, USA) was used for all the analyses; the significance was set at $p<0.05$.

\section{Results}

\section{Anatomical evaluation}

There were significant positive correlations between the screw diameter and \%fill $(r=0.69, p<0.01)$ and screw length and \%length $(r=0.84, p<0.01)$ (Table 1$)$. The value of \%fill as well as the difference between the minor endosteal width and the outer screw diameter were $59.6 \% \pm 10.9 \%$ and $3.1 \pm 1.4 \mathrm{~mm}$ with $4.5-\mathrm{mm}$ diameter screws, $72.8 \% \pm 13.4 \%$ and $2.1 \pm 1.4 \mathrm{~mm}$ using $5.5-\mathrm{mm}$ diameter screws, $86.1 \% \pm 15.9 \%$ and $1.1 \pm 1.4 \mathrm{~mm}$ using 6.5 $\mathrm{mm}$ diameter screws, and $99.3 \% \pm 18.3 \%$ and $0.1 \pm 1.4 \mathrm{~mm}$ using 7.5-mm diameter screws.

\section{Correlation between bone mineral density and the fixation parameters}

Among the BMD parameters, femoral neck BMD, measured using DEXA, showed the strongest correlation with the fixation parameters except for the vertebral fixation strength against axial rotation and the equivalent stress of the bone-screw interface (Table 2).

\section{Pullout strength}

Compared to screws with a length of $40 \mathrm{~mm}$, the pullout

Table 1. Anatomical parameters

\begin{tabular}{|c|c|}
\hline Variable & Value \\
\hline \multicolumn{2}{|c|}{ Screw diameter (mm) } \\
\hline 4.5 & $59.6 \pm 10.9$ \\
\hline 5.5 & $72.8 \pm 13.4$ \\
\hline 6.5 & $86.1 \pm 15.9$ \\
\hline 7.5 & $99.3 \pm 18.3$ \\
\hline \multicolumn{2}{|c|}{ Screw length (mm) } \\
\hline 30 & $29.2 \pm 5.5$ \\
\hline 35 & $41.7 \pm 5.6$ \\
\hline 40 & $55.7 \pm 5.2$ \\
\hline 45 & $68.1 \pm 6.2$ \\
\hline 50 & $81.1 \pm 5.9$ \\
\hline
\end{tabular}

Values are presented as mean \pm standard deviation.

Table 2. Correlation coefficient between bone mineral density and fixation parameters

\begin{tabular}{lccccc} 
& \multicolumn{3}{c}{ Dual-energy X-ray absorptiometry } & & \multicolumn{2}{c}{ Hounsfield units } \\
\cline { 2 - 5 } Variable & Femoral neck & Mean lumbar & L4 & Pedicle & Vertebral body \\
\hline Pullout strength & $0.47^{* *}$ & 0.07 & $0.28^{* *}$ & $0.42^{* *}$ & $0.30^{* *}$ \\
\hline Vertebral fixation strength & & & & $0.23^{*}$ & $0.23^{*}$ \\
\hline Flexion & $0.39^{* *}$ & 0.02 & 0.13 & $0.25^{* *}$ & $0.19^{*}$ \\
\hline Extension & $0.38^{* *}$ & 0.02 & 0.17 & $0.48^{* *}$ & $0.39^{* *}$ \\
\hline Lateral bending & $0.49^{* *}$ & $0.31^{* *}$ & 0.04 & 0.11 & $0.41^{* *}$ \\
\hline Axial rotation & $0.36^{* *}$ & 0.18 & $0.25^{* *}$ & $0.63^{* *}$ & $0.46^{* *}$ \\
\hline Equivalent stress & $0.38^{* *}$ & $0.22^{*}$ & 0.05 & &
\end{tabular}

${ }^{*} p<0.05$. ${ }^{* *} p<0.05$. 


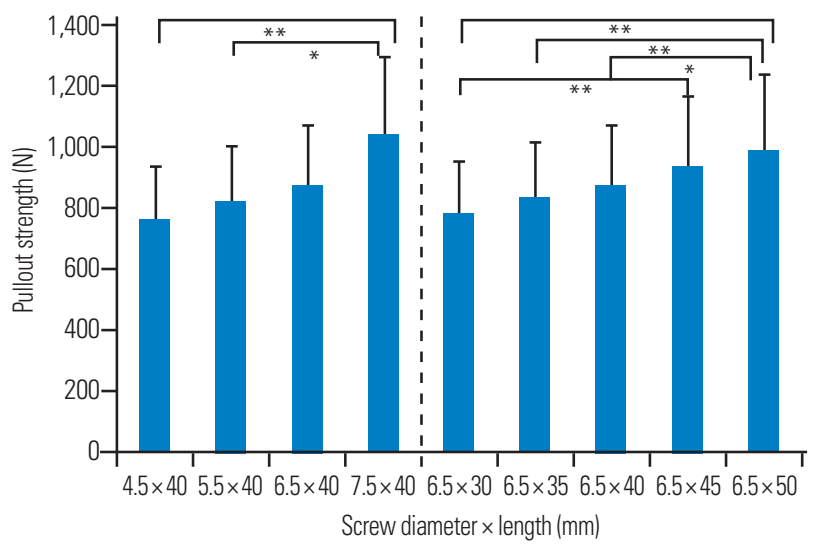

Fig. 3. Comparison of the mean pullout strength. The pullout strength increases significantly with increasing screw diameter (left) and length (right). Significant at ${ }^{*} p<0.05$. Significant at ${ }^{* *} p<0.01$.

Table 3. Multiple regression analysis of each fixation parameter

\begin{tabular}{|c|c|c|c|}
\hline Variable & Category & $\begin{array}{l}\text { Significant } \\
\text { independent } \\
\text { factors }(\boldsymbol{B})\end{array}$ & $R^{2}$ \\
\hline \multirow[t]{4}{*}{ Pullout strength } & & & 0.45 \\
\hline & Femoral BMD & 0.47 & \\
\hline & Diameter & 0.38 & \\
\hline & Length & 0.29 & \\
\hline \multicolumn{4}{|c|}{ Vertebral fixation strength } \\
\hline \multirow[t]{4}{*}{ Flexion } & & & 0.45 \\
\hline & Femoral BMD & 0.45 & \\
\hline & Diameter & 0.43 & \\
\hline & \%Length & 0.23 & \\
\hline \multirow[t]{4}{*}{ Extension } & & & 0.44 \\
\hline & Femoral BMD & 0.43 & \\
\hline & Diameter & 0.42 & \\
\hline & \%Length & 0.26 & \\
\hline \multirow[t]{4}{*}{ Lateral bending } & & & 0.42 \\
\hline & Femoral BMD & 0.46 & \\
\hline & Diameter & 0.17 & \\
\hline & \%Length & 0.25 & \\
\hline \multirow[t]{4}{*}{ Axial rotation } & & & 0.43 \\
\hline & Vertebral body CT & 0.40 & \\
\hline & Diameter & 0.22 & \\
\hline & \%Length & 0.36 & \\
\hline \multirow[t]{4}{*}{ Equivalent stress } & & & 0.68 \\
\hline & Pedicle CT & 0.58 & \\
\hline & Diameter & -0.24 & \\
\hline & \%Length & -0.42 & \\
\hline
\end{tabular}

$\beta$, standard regression coefficient; $R^{2}$, coefficient of determination; BMD, bone mineral density; $C$, computed tomography. strength was significantly higher for screws with a larger diameter $(p<0.01)$ (Fig. 3). Compared to screws with a diameter of $6.5 \mathrm{~mm}$, the pullout strength was significantly higher for longer screws $(p<0.01)$. All size parameters (screw diameter, \%fill, screw length, and \%length) were significantly correlated with pullout strength; among these, the screw diameter $(r=0.41, p<0.01)$ and screw length $(r=0.53, p<0.01)$ affected the pullout strength significantly more than \%fill $(r=0.34, p<0.01)$ and \%length $(r=0.51, p<0.01)$. Best-subset multiple regression analysis showed that femoral neck BMD (standardized regression coefficient $[\beta]=0.47, p<0.01)$, screw diameter $(\beta=0.38$, $p<0.01)$, and screw length $(\beta=0.29, p<0.01)$ were significant independent factors $\left(R^{2}=0.45, p<0.01\right)$ (Table 3$)$. Residual analysis showed no violations of the assumptions of normality, linearity, and homoscedasticity.

Table 4. Vertebral fixation strength $(\mathrm{N} / \mathrm{mm})$

\begin{tabular}{|lllll}
\hline Screw size & Flexion & Extension & Lateral bending & Axial rotation \\
\hline$\Phi 4.5 \times 40 \mathrm{~mm}$ & $457 \pm 99$ & $441 \pm 126$ & $1,823 \pm 724$ & $2,774 \pm 1,866$ \\
\hline$\Phi 5.5 \times 40 \mathrm{~mm}$ & $480 \pm 119$ & $481 \pm 157$ & $1,833 \pm 751$ & $3,111 \pm 1,289$ \\
\hline$\Phi 6.5 \times 40 \mathrm{~mm}$ & $588 \pm 175$ & $605 \pm 182$ & $1,951 \pm 692$ & $3,232 \pm 1,865$ \\
\hline$\Phi 7.5 \times 40 \mathrm{~mm}$ & $804 \pm 199$ & $817 \pm 261$ & $2,836 \pm 960$ & $3,834 \pm 1,120$ \\
\hline$\Phi 6.5 \times 30 \mathrm{~mm}$ & $579 \pm 138$ & $563 \pm 80$ & $1,770 \pm 836$ & $2,792 \pm 765$ \\
\hline$\Phi 6.5 \times 35 \mathrm{~mm}$ & $583 \pm 154$ & $593 \pm 209$ & $1,846 \pm 864$ & $3,026 \pm 961$ \\
\hline$\Phi 6.5 \times 45 \mathrm{~mm}$ & $635 \pm 166$ & $638 \pm 140$ & $2,598 \pm 837$ & $3,979 \pm 1,784$ \\
\hline $66.5 \times 50 \mathrm{~mm}$ & $667 \pm 151$ & $692 \pm 192$ & $2,636 \pm 886$ & $4,450 \pm 2,289$ \\
\hline
\end{tabular}

Values are presented as mean \pm standard deviation.

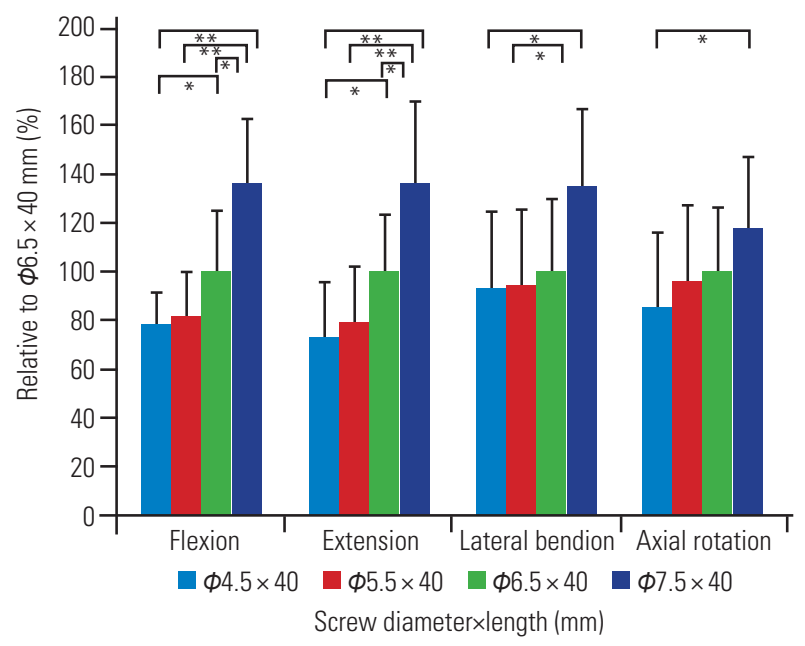

Fig. 4. Comparison of fixation strength among different diameters of screws. The fixation strength of a $6.5 \times 40-\mathrm{mm}$ screw was set to $100 \%$. The strength increases significantly with increasing diameter. Significant at ${ }^{*} p<0.05$. Significant at ${ }^{* *} p<0.01$. 


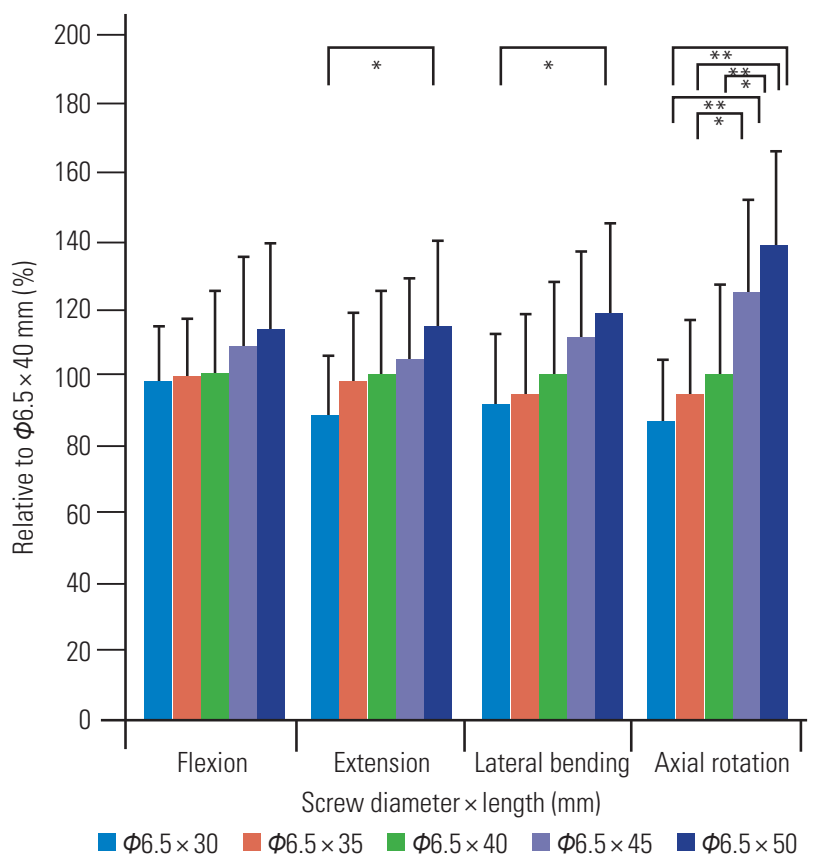

Fig. 5. Comparison of fixation strength among different lengths of screws. The fixation strength of a $6.5 \times 40-\mathrm{mm}$ screw was set to $100 \%$. The strength increases significantly with increasing length, except on flexion. Significant at * $p<0.05$. Significant at ${ }^{* *} p<0.01$.

\section{Vertebral fixation strength}

The ultimate failure load and vertebral fixation strength rose significantly with an increase in the screw diameter and length in each plane of motion (Table 4 and Figs. 4, $5)$. As per the multiple regression analyses, screw diameter and \%length had a significantly higher impact on the vertebral fixation strength as compared to the \%fill and screw length in each plane of motion (Table 3). For flexion and extension moments, the influence of screw diameter $(\beta=0.42-0.43)$ was greater than that of \%length $(\beta=0.23-0.26)$, and for lateral bending and axial rotation, the influence of \%length $(\beta=0.25-0.36)$ was greater than that of the screw diameter $(\beta=0.17-0.22)$.

\section{Equivalent stress of bone-screw interface}

The equivalent stress decreased significantly with an increase in both, the screw diameter and screw length (Fig. 6). Multiple regression analyses showed that the $\mathrm{HU}$ values of the pedicle $(\beta=0.58, p<0.01)$, screw diameter $(\beta=-0.24, p<0.05)$, and \%length $(\beta=-0.42, p<0.01)$ were significant independent factors that influenced the equivalent stress of the bone-screw interface $\left(R^{2}=0.68, p<0.01\right)$ (Table 3).
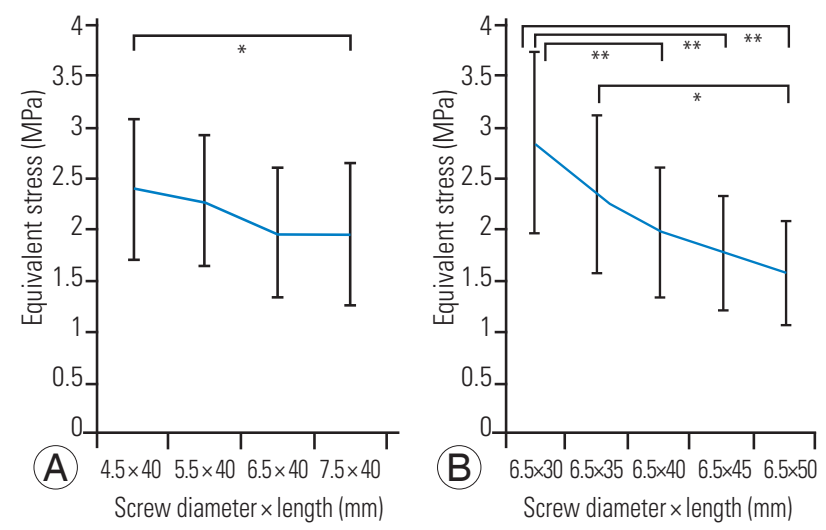

Fig. 6. (A, B) Comparison of mean equivalent stress of bone-screw interface among different sizes of screws. The stress value decreases significantly with increasing screw diameter (left) and length (right). Significant at ${ }^{*} p<0.05$. Significant at ${ }^{* *} p<0.01$

\section{Discussion}

The present study quantitatively determined the impact of screw size on screw fixation in osteoporotic vertebrae using computational simulation. Screw diameter had a stronger impact on the resistance to screw pullout and flexion-extension loading. Screw depth in the vertebra (\%length) had a greater impact on the resistance to lateral bending and axial rotation loading and mechanical stress of the bone-screw interface. Contrary to other testing methods that use polyurethane foams, animal models, and cadaveric spines, the FE method can facilitate fair and reproducible comparisons in the same environmental conditions, while lowering the expense, time, and effort that is required for repeated mechanical tests of large samples [3,4,10,13-15].

As per the multiple regression analyses, BMD parameters were the most critical factors that influenced various screw fixations; this finding was in agreement with previous reports $[1,6,7]$. It is noteworthy that femoral neck BMD showed the strongest correlation among the three types of BMD parameters measured using DEXA. The present study enrolled patients with lumbar degeneration; the presence of osteophyte formation, facet hypertrophy, and aortic calcification may affect the lumbar BMD data and overestimate its true value $[14,19]$.

In terms of the pullout strength, screw diameter and length were identified as predictors, with the effect of screw diameter being more remarkable than that of screw length. Although many researchers have stressed on the importance of a larger screw diameter to contact denser cortical bone within the pedicle $[2,9,10]$, our study 
showed that the actual screw diameter exerted a stronger effect on the pullout strength as compared to screw fitness in the pedicle (\%fill). This could be attributed to the anatomical characteristics of the pedicle that is the most important part of pedicle screw fixation. Hirano et al. [11] investigated the detailed structure of the pedicle with quantitative $\mathrm{CT}$ and showed that the cortical layer of the pedicle in the osteoporotic vertebrae was 70\% thinner and $17 \%$ weaker than that in normal vertebrae. We created FE models of the L4 vertebra that are high demand clinically, with a mean \%fill in the pedicle of $99.3 \%$ (the mean difference between the minor endosteal width and the outer screw diameter: $0.1 \mathrm{~mm}$ ) using $7.5-\mathrm{mm}$-diameter screws. The endosteal width of the L4 pedicle isthmus in this study was $7.6 \pm 1.1 \mathrm{~mm}$; this was relatively close to $5.7-7.5$ $\mathrm{mm}$ reported in previous studies [20,21]. Li et al. [20] recommended that the maximum screw diameter was the pedicle isthmus endosteal width $+1 \mathrm{~mm}$ to prevent pedicle fracture. Thus, we assumed that a larger diameter screw may have a different effect on screw fixation owing to increased cortical bone contact in the pedicle in a future study. With respect to the effect of screw length on fixation, Zindrick et al. [2] and Karami et al. [22] concluded that the pullout strength increased with longer screws, but only improved when the screws were placed deep enough to penetrate the anterior cortex. Weinstein et al. [17] reported that the pullout strength of pedicle screws in normal vertebrae was responsible for $60 \%$ of the fixation at the pedicle and $15 \%-20 \%$ at the cancellous bone in the vertebral body. While the progression of osteoporosis weakens the cancellous bone structure within the vertebrae, longer screws increase the degree of bone contact; this may have contributed slightly to the increased pullout strength even without engagement with the anterior cortex in the present study.

Comparisons of the vertebral fixation strength of the paired pedicle screw construct showed that screw diameter and \%length were contributing factors. There are individual differences in the vertebral morphology and size; therefore, we considered that the depth of screw insertion was more important to hold the vertebral body than the actual screw length. To our knowledge, few studies have been reported on the effect of screw insertion depth on vertebral fixation in osteoporotic vertebrae. In normal vertebrae, Krag et al. [23] examined the effect of screw insertion depth under flexion and torsion loads and reported that increasing the screw depth from 50\% to $80 \%$ increased the fixation by about 30\%. Similarly, Bezer et al. [24] compared a lateral directional bicortical screw (average screw length, $36.3 \mathrm{~mm}$ ) with a conventional monocortical screw (average screw length, $43.1 \mathrm{~mm}$ ) and demonstrated that the rotational stability was $23.9 \%$ more for the conventional screw even without bicortical purchase. With respect to the importance of screw diameter, Hirano et al. [11] reported that the pedicular portion contributed $80 \%$ to the caudocephalad stiffness of pedicle screws. In agreement with this result, our findings showed that the effect of screw diameter against flexion and extension motion was more marked than the effect of the \%length.

Finally, with respect to the equivalent stress of the bonescrew interface, screw diameter and \%length, not the actual screw length, were identified as significant predictive factors for effective distribution of the load applied on the vertebrae. Although caudocephalad cyclic loading is known to be important for the mechanism of pedicle screw loosening [25], it was difficult to simulate the effect of cyclic loading using the current FE models. Therefore, we considered that the equivalent stress value of the bonescrew interface may influence long-term screw loosening [14]. Zindrick et al. [2] stated that deeper screw insertion caused greater resistance against cyclic loading; this report supported our findings.

The present findings have practical implications because few studies have quantitatively investigated the effect of screw size on fixation in osteoporotic vertebrae, while the pedicle screw technique has become the gold standard for spinal arthrodesis. Screw size should be determined, not uniformly or by surgeon's preference, but by considering the type of mechanical force applied to the corresponding vertebrae as well as the anatomical limitation, such as the risks of pedicle fracture and neurovascular injury. The proximal and distal levels of the construct are prone to screw loosening or backout; therefore, deeper screw insertion and the use of a larger diameter screw are reasonable for the maintenance of stability until adequate bone arthrodesis is achieved. In order to apply the rotational force successfully for apical vertebrae located at the apex of the curve, the screws should be placed as deep as possible into the vertebral column, sufficiently passing the instantaneous axis of rotation. One concern regarding deeper screw placement is a high risk of critical vascular and visceral injuries in front of the vertebral body that warrants fluoroscopic or navigation assistance for appropriate screw placement. 
This study used FE analysis and has intrinsic limitations. First, the testing protocol used for the FE models of the single vertebral segment in the present study did not perfectly reflect the physiological loads acting on the vertebrae in vivo; further research and experimental validation is required on this subject. Although the multiplesegment construct model represents a more realistic clinical situation, it requires complex modeling, such as material and geometric properties of the intervertebral elements, potentially leading to more complicated results. In contrast, the models used in this study could provide results that directly reflected the holding power of the placed screws [14]. Second, the coefficient of determination of the multiple regression equations were $R^{2}=0.42-$ 0.68 that limits the use as prediction equations for various screw fixations. However, to our knowledge, no report has quantitatively and comprehensively examined screw fixation in osteoporotic vertebrae; thus, we believe that the results are useful.

\section{Conclusions}

In this study, we determined the effect of screw size on screw fixation in osteoporotic vertebrae. The screw size should be determined as per the biomechanical behavior of the screws, type of mechanical force applied to the corresponding vertebra, and anatomical limitations.

\section{Conflict of Interest}

No potential conflict of interest relevant to this article was reported.

\section{Author Contributions}

Conception and design: all authors; data acquisition: all authors; analysis of data: Keitaro Matsukawa; drafting of the manuscript: Keitaro Matsukawa; critical revision: Yoshiyuki Yato, Hideaki Imabayashi; obtaining funding: none funding; administrative support: Yoshiyuki Yato, Hideaki Imabayashi; and supervision: Yoshiyuki Yato.

\section{References}

1. Halvorson TL, Kelley LA, Thomas KA, Whitecloud TS 3rd, Cook SD. Effects of bone mineral density on pedicle screw fixation. Spine (Phila Pa 1976)
1994;19:2415-20.

2. Zindrick MR, Wiltse LL, Widell EH, et al. A biomechanical study of intrapeduncular screw fixation in the lumbosacral spine. Clin Orthop Relat Res 1986;(203):99-112.

3. Hsu CC, Chao CK, Wang JL, Hou SM, Tsai YT, Lin J. Increase of pullout strength of spinal pedicle screws with conical core: biomechanical tests and finite element analyses. J Orthop Res 2005;23:788-94.

4. Chao CK, Hsu CC, Wang JL, Lin J. Increasing bending strength and pullout strength in conical pedicle screws: biomechanical tests and finite element analyses. J Spinal Disord Tech 2008;21:130-8.

5. Chatzistergos PE, Sapkas G, Kourkoulis SK. The influence of the insertion technique on the pullout force of pedicle screws: an experimental study. Spine (Phila Pa 1976) 2010;35:E332-7.

6. Cho W, Cho SK, Wu C. The biomechanics of pedicle screw-based instrumentation. J Bone Joint Surg Br 2010;92:1061-5.

7. Weiser L, Huber G, Sellenschloh K, et al. Insufficient stability of pedicle screws in osteoporotic vertebrae: biomechanical correlation of bone mineral density and pedicle screw fixation strength. Eur Spine J 2017;26:2891-7.

8. Kim YY, Choi WS, Rhyu KW. Assessment of pedicle screw pullout strength based on various screw designs and bone densities: an ex vivo biomechanical study. Spine J 2012;12:164-8.

9. Soshi S, Shiba R, Kondo H, Murota K. An experimental study on transpedicular screw fixation in relation to osteoporosis of the lumbar spine. Spine (Phila Pa 1976) 1991;16:1335-41.

10. Bianco RJ, Arnoux PJ, Wagnac E, Mac-Thiong JM, Aubin CE. Minimizing pedicle screw pullout risks: a detailed biomechanical analysis of screw design and placement. Clin Spine Surg 2017;30:E226-32.

11. Hirano T, Hasegawa K, Takahashi HE, et al. Structural characteristics of the pedicle and its role in screw stability. Spine (Phila Pa 1976) 1997;22:2504-9.

12. Lai DM, Shih YT, Chen YH, Chien A, Wang JL. Effect of pedicle screw diameter on screw fixation efficacy in human osteoporotic thoracic vertebrae. J Biomech 2018;70:196-203.

13. Chen SI, Lin RM, Chang CH. Biomechanical investigation of pedicle screw-vertebrae complex: a finite element approach using bonded and contact interface 
conditions. Med Eng Phys 2003;25:275-82.

14. Matsukawa K, Yato Y, Imabayashi H, et al. Biomechanical evaluation of fixation strength among different sizes of pedicle screws using the cortical bone trajectory: what is the ideal screw size for optimal fixation? Acta Neurochir (Wien) 2016;158:465-71.

15. Matsuura Y, Giambini H, Ogawa Y, et al. Specimenspecific nonlinear finite element modeling to predict vertebrae fracture loads after vertebroplasty. Spine (Phila Pa 1976) 2014;39:E1291-6.

16. Keyak JH, Rossi SA, Jones KA, Skinner HB. Prediction of femoral fracture load using automated finite element modeling. J Biomech 1998;31:125-33.

17. Weinstein JN, Rydevik BL, Rauschning W. Anatomic and technical considerations of pedicle screw fixation. Clin Orthop Relat Res 1992;(284):34-46.

18. Brantley AG, Mayfield JK, Koeneman JB, Clark KR. The effects of pedicle screw fit: an in vitro study. Spine (Phila Pa 1976) 1994;19:1752-8.

19. Buhler DW, Berlemann U, Oxland TR, Nolte LP. Moments and forces during pedicle screw insertion: in vitro and in vivo measurements. Spine (Phila $\mathrm{Pa}$ 1976) 1998;23:1220-7.

20. Li B, Jiang B, Fu Z, Zhang D, Wang T. Accurate determination of isthmus of lumbar pedicle: a mor- phometric study using reformatted computed tomographic images. Spine (Phila Pa 1976) 2004;29:243844.

21. Makino T, Kaito T, Fujiwara H, Yonenobu K. Analysis of lumbar pedicle morphology in degenerative spines using multiplanar reconstruction computed tomography: what can be the reliable index for optimal pedicle screw diameter? Eur Spine J 2012;21:1516-21.

22. Karami KJ, Buckenmeyer LE, Kiapour AM, et al. Biomechanical evaluation of the pedicle screw insertion depth effect on screw stability under cyclic loading and subsequent pullout. J Spinal Disord Tech 2015;28:E133-9.

23. Krag MH, Beynnon BD, Pope MH, DeCoster TA. Depth of insertion of transpedicular vertebral screws into human vertebrae: effect upon screw-vertebra interface strength. J Spinal Disord 1988;1:287-94.

24. Bezer M, Ketenci IE, Saygi B, Kiyak G. Bicortical versus unicortical pedicle screws in direct vertebral rotation: an in vitro experimental study. J Spinal Disord Tech 2012;25:E178-82.

25. Law M, Tencer AF, Anderson PA. Caudo-cephalad loading of pedicle screws: mechanisms of loosening and methods of augmentation. Spine (Phila Pa 1976) 1993;18:2438-43. 